\title{
Effect of a Concurrent Well-Rounded Exercise Training Using a Floor-Based Exercise Station in Older Women
}

\author{
Aiko Imai, ${ }^{1,2}$ Naoko Sengoku1, Daisuke Koizumi', Yukiko Kitabayashi', \\ Aiko Naruse' ${ }^{1}$ Michael E. Rogers ${ }^{3}$ and Nobuo Takeshima ${ }^{1,4}$ \\ ${ }^{1}$ Active Aging Association, Kitaku, Nagoya 462-0828, JAPAN \\ e-mail: takeshima@alice.asahi-u.ac.jp \\ ${ }^{2}$ Department of Rehabilitation, Seijyo University, Tokai 476-0014, JAPAN \\ ${ }^{3}$ Department of Human Performance Studies, Center for Physical Activity and Aging, Wichita State University, \\ Wichita, KS 67260-0016, USA \\ ${ }^{4}$ Department of Health and Sports Sciences, School of Health Sciences, Asahi University, Mizuho, Gifu 501-0296, JAPAN \\ [Received January 30, 2017; Accepted November 16, 2017; Published online December 1, 2017]
}

\begin{abstract}
To evaluate the effects of a 12-wk circuit exercise training program using the floor-based exercise station (FBES) on aerobic fitness, strength, and balance in older women. Participants were divided into: FBES exercise group ( $\mathrm{EX}: \mathrm{n}=22 ; 68.1 \pm 6.5 \mathrm{yr}$ ) and a non-exercise control group $(C N: n=18 ; 68.2 \pm 5.7 \mathrm{yr})$. EX participated in a 12-wk circuit training program, $3 \mathrm{~d} / \mathrm{wk}$ for 50 $\mathrm{min} / \mathrm{d}$, consisting of warm-up exercise $(10 \mathrm{~min})$, circuit training $(30 \mathrm{~min})$, and cool-down/relaxation exercise $(10 \mathrm{~min})$. Twelve strength and balance exercises and 12 aerobic dance exercises were performed alternatively for 30s each with a heart rate of 100-110 bpm. CN continued normal physical activity patterns. After 12-wk, all measurements were repeated in both groups. Compared to CN, EX increased $(p<0.05)$ arm curl $(12.6 \%)$, timed up-and-go $(-8.7 \%)$, 12-min walk (5.4\%), predict $\dot{\mathbf{V}} \mathrm{O}_{2} \max (6.3 \%)$, sit-and-reach $(24.3 \%)$, and back scratch $(271.5$ $\%)$, but not balance parameters. Incorporating both aerobic and resistance training via the FBES improves multiple aspects of fitness but not balance. These results are similar to those from larger, more expensive hydraulic exercise machines. This study supports the efficacy of the FBES for older adults and implementation is feasible in multiple settings as it is relatively inexpensive and requires little space.
\end{abstract}

Keywords: circuit exercise, concurrent exercise, older adults

\section{Introduction}

Each component of physical fitness (i.e., cardiorespiratory fitness, muscular strength and endurance (muscular fitness), body composition, flexibility and neuromotor (balance)) should be addressed in an exercise program for older adults (American College of Sports Medicine, 2009; 2011) as benefits can be gained from each individual mode of exercise (Wood et al., 2001; Izquierdo et al., 2004) and a well-rounded exercise (WRE) program consisting of multiple modes of exercise (aerobic, resistance, flexibility and balance) can be effective in improving the components of functional fitness in older adults (NIA 1996; Takeshima et al., 2007). However, it can be challenging to commit the time to perform- ing the recommended amounts of exercise for each mode of exercise on a weekly basis. Therefore, concurrent exercise, or combining multiple modes into a single session, may be a more practical and beneficial approach.

Circuit training is one approach to combining multiple modes of exercise. Circuit training using hydraulic resistance machines (PACE training) elicits significant improvements in cardio-respiratory fitness, muscular fitness, body composition (decrease of subcutaneous fat), and high-density lipoprotein cholesterol (HLC) (Takeshima et al., 2004; Yamauchi et al., 2005). PACE training is a safe and useful method for providing concurrent exercise for older people but such programs do not include balance exercises that are an important 
activity for fall prevention. Other limitations to PACE training are that it entails high costs because the pneumatic devices are expensive and they require a large amount of permanently dedicated space the size of a gymnasium or public hall.

Another type of concurrent exercise is Nordic walking which is an outdoor activity involving specially designed poles used to push against the ground with each stride for the purpose of activating the upper body while walking. Nordic walking has been shown to improve cardiovascular fitness and muscle strength in older adults, but not balance ability (Takeshima et al., 2013). Although this activity is easy to perform once a person is trained to properly use the poles, it does require outdoor space and does not appear to affect static balance and dynamic balance (Takeshima et al., 2013).

Ideally, a concurrent exercise program for older adults should be effective, inexpensive, and require little space. Recently, some new devices that a floorbased exercise station (FBES) for concurrent exercise have appeared on the market in the world. One such product is the Thera-Band Exercise Station (Performance Health, Akron, $\mathrm{OH}$ ) that was designed to combine strength, balance and flexibility exercises into one convenient system for fitness. This device is a platform with six connection points allowing elastic resistance tubing to be attached at a variety of locations and a center space to allow the placement of an exercise ball or foam pads for balance training (Figure 1). This device is relatively small and flat $\left(44^{\prime \prime} \times 24^{\prime \prime} \times 2^{\prime \prime}\right)$ so the devices can be stacked and stored easily. Therefore, community centers could provide temporary space to offer a class utilizing many of these devices and later store them in a closet, or allow a person to exercise at home and store it under a bed. However, there are no studies demonstrating the efficacy of concurrent

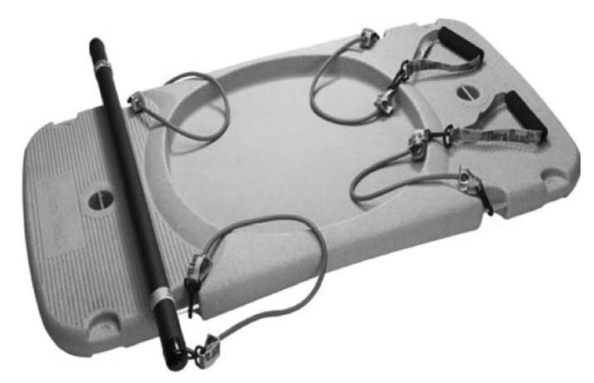

Figure 1 Thera-Band Exercise Station. exercise programs utilizing of the FBS in older adults.

The aim of this study was to evaluate the effects of a 12-wk community center-based circuit exercise training program using the FBES on fitness in older women. It was hypothesized that the program would improve strength, balance, flexibility, and cardiorespiratory fitness in the exercise group compared to a control group.

\section{Methods}

\subsection{Subjects}

In response to a local newspaper advertisement, 43 older women from Nagoya, Japan, volunteered to participate in this study. Prior to acceptance into the study, a physician conducted a medical examination on each participant that included a questionnaire regarding medical history and evaluation of elektrokardiogramm (EKG) and blood pressure during an exercise test. Based on contraindicative responses during the exercise test, three volunteers were excluded.

Twenty-two community-dwelling volunteers were randomly placed in the circuit exercise group (EX: age $=68.1 \pm 6.5$ years; body height $=151.0 \pm 5.0 \mathrm{~cm}$; mass $=49.4 \pm 5.5 \mathrm{~kg}$ (mean $\pm \mathrm{SD})$ ). An additional 21 community-dwelling volunteers were randomly placed in the control group but 3 participants did not return for post-testing so they were not included in the analysis $(\mathrm{CN}$ : age $=68.2 \pm 5.7$ years; body height $=152.3 \pm 4.3 \mathrm{~cm} ; \quad$ mass $=50.6 \pm 6.6 \mathrm{~kg}$ ). A slightly greater number of people were assigned to EX than $\mathrm{CN}$ to account for the greater potential for attrition in EX. There were no significant differences in the physical characteristics between the groups at baseline (Table 1).

The ethical committee of Nagoya City University approved the study. All participants received written and oral instructions for the study and each gave their written informed consent prior to participation.

\subsection{Exercise program}

Circuit exercise training was conducted over a 12 -wk period $(3 \mathrm{~d} / \mathrm{wk}, 50 \mathrm{~min} /$ day; $10 \mathrm{~min}$ of warm-up, $30 \mathrm{~min}$ of circuit exercise, and $10 \mathrm{~min}$ of cool-down) at a Nagoya City University laboratory 
Table 1 Comparison of physical characteristics and fitness parameters between groups at pre-test.

\begin{tabular}{|c|c|c|c|}
\hline & $\begin{array}{l}\text { Exercise (EX) group } \\
(\mathrm{n}=22)\end{array}$ & $\begin{array}{l}\text { Non-exercise }(\mathrm{CN}) \text { group } \\
(\mathrm{n}=18)\end{array}$ & $\begin{array}{c}\text { Comparison between } \\
\text { both groups }\end{array}$ \\
\hline Age (year) & $68.1 \pm 6.5$ & $68.2 \pm 5.7$ & N.S. \\
\hline Height (cm) & $151.0 \pm 5.0$ & $152.3 \pm 4.3$ & N.S. \\
\hline Body Mass (kg) & $49.4 \pm 5.5$ & $50.6 \pm 6.6$ & N.S. \\
\hline BMI $\left(\mathrm{kg} / \mathrm{m}^{2}\right)$ & $21.8 \pm 2.2$ & $22.0 \pm 2.2$ & N.S. \\
\hline$\%$ fat $(\%)$ & $29.5 \pm 3.8$ & $28.5 \pm 3.7$ & N.S. \\
\hline Fat (kg) & $14.7 \pm 3.0$ & $14.6 \pm 3.3$ & N.S. \\
\hline LBM (kg) & $34.7 \pm 3.4$ & $36.4 \pm 4.1$ & N.S. \\
\hline $\mathrm{AC}($ times $/ 30 \mathrm{sec})$ & $26.2 \pm 3.7$ & $25.7 \pm 5.0$ & N.S. \\
\hline CS (times/30 sec) & $28.9 \pm 5.5$ & $27.3 \pm 6.0$ & N.S. \\
\hline UPGO (sec) & $4.35 \pm 0.56$ & $4.24 \pm 0.62$ & N.S. \\
\hline $\mathrm{SR}(\mathrm{cm})$ & $18.1 \pm 9.1$ & $19.6 \pm 8.4$ & N.S. \\
\hline $\mathrm{BS}(\mathrm{cm})$ & $0.7 \pm 7.9$ & $-1.5 \pm 13.1$ & N.S. \\
\hline FR $(\mathrm{cm})$ & $32.3 \pm 5.0$ & $31.9 \pm 2.9$ & N.S. \\
\hline TW (m) & $1162 \pm 112$ & $1131 \pm 83$ & N.S. \\
\hline Predict $\dot{\mathrm{V}} \mathrm{O}_{2} \max$ & $23.8 \pm 2.7$ & $22.7 \pm 2.4$ & N.S. \\
\hline SVcomp (deg/sec) & $0.82 \pm 0.25$ & $0.81 \pm 0.18$ & N.S. \\
\hline EPEcomp (\%) & $78.6 \pm 12.4$ & $78.0 \pm 8.1$ & N.S. \\
\hline MXEcomp (\%) & $93.4 \pm 5.6$ & $92.4 \pm 4.0$ & N.S. \\
\hline
\end{tabular}

Note: BMI: body mass index (BMI) was computed as body weight $(\mathrm{kg})$ divided by the square of height (m); LBM: lean body mass; AC: arm curl; CS: Chair stand; UPGO: Up \& go; SR: Sit \& reach; BS: Back scratch; FR: Functional Reach; TW: 12-min walk; Predict $\dot{\mathrm{V}} \mathrm{O}_{2} \max$ : $\dot{\mathrm{V}} \mathrm{O}_{2} \max$ was caluculate by equation from 12-min walk; SVcomp: Composite sway velocity scores were calculated based on each sway velocity condition; EPEcomp: Composite Endpoint excursion scores were calculated based on movments toward all 8 targets appeared around a center square at 0, 45, 90, 135, 180, 225, 270, and 315 degrees; MXE: Composite maximum excursion scores were calculated based on movments toward all 8 targets appeared around a center square at $0,45,90,135,180,225$, 270, and 315 degrees; N.S., not significant.

(Figure 2). Each session was led by trained fitness instructors and was supervised by the researchers. Music with a tempo of $120-130 \mathrm{~b} / \mathrm{min}$ was played while performing the circuit exercise. Exercise intensity was monitored based on heart rate (HR) using a HR monitor (Aqurexplus, Polar, Finland) and subjective ratings of perceived exertion (RPE) (Borg, 1982) were recorded after each exercise. The exercise intensity was set at $60-70 \%$ of individual heart rate reserve (HRR) (Karvonen et al., 1957) during exercise. An instructor prompted participants to increase the intensity if they did not reach the target heart rate.

The warm-up and cool-down activities consisted of relaxation activities using breathing techniques and stretching exercises performed slowly with each position was held for $20 \mathrm{sec}$. The participants were asked to stretch to the point where they felt moderate tension without feeling pain in joints or muscles.

The circuit exercise program consisted of a series of 24 stations to perform aerobic, resistance, and balance exercises. Participants performed a specific exercise at each station for $30 \mathrm{sec}$ and then moved to the next station until 30 min of circuit training had been completed. Eight FBES units had elastic resistance tubing clipped to them for strength training of all major muscle groups. Inflated exercise balls (diameter $=55 \mathrm{~cm}$ ) or foam pads (Stability Trainers, Performance Health, Akron, $\mathrm{OH}$ ) were placed on four additional FBES units for balance training. 

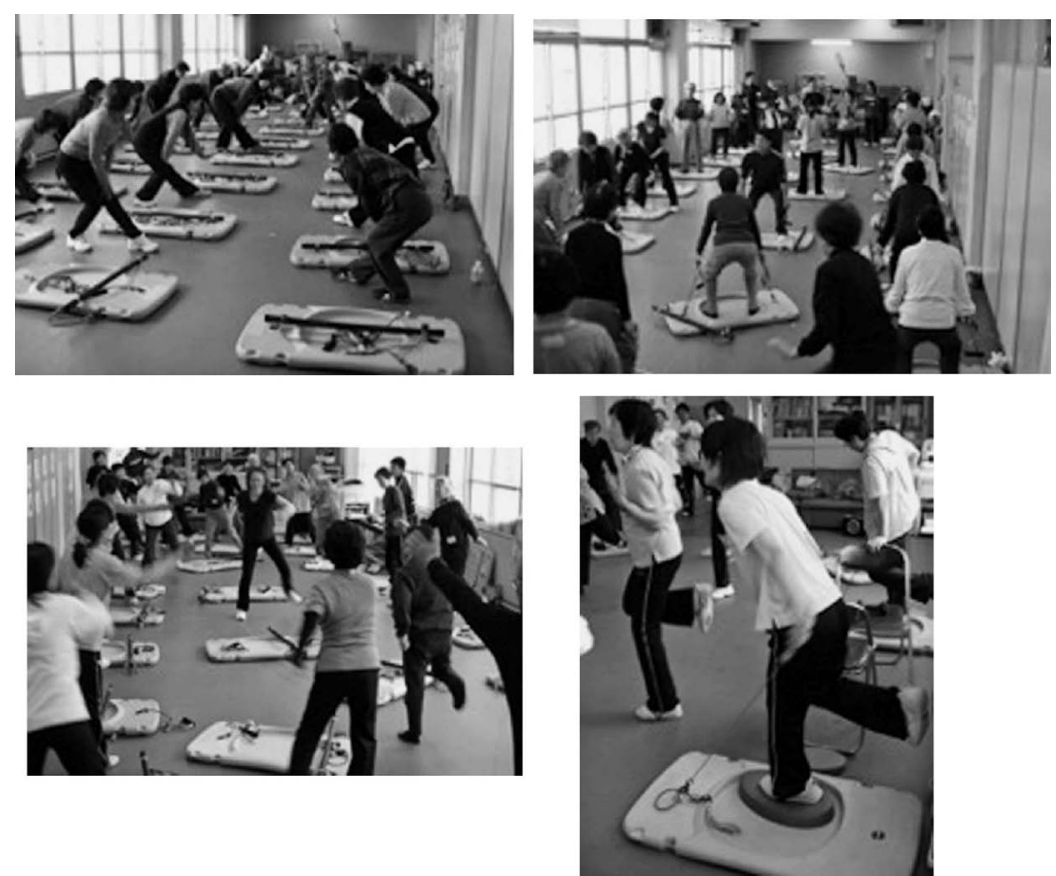

Figure 2 FBES curcuit training program.

Twelve aerobic dancing boards were placed between each FBES unit in a circular manner to perform the circuit training. This design was similar to our previously-evaluated PACE circuit exercise program that utilized hydraulic resistance machines (Takeshima et al., 2004).

The program was divided into three monthly stages. Participants recorded exercise intensities during each exercise and the relative intensity for all exercises increased progressively each month. The aerobic dance stations consisted of marching in place and raising the arms for $30 \mathrm{sec}$. The eight resistance exercises were arm curl, squat, upright rowing, lateral raise, elbow extension, low back extension, trunk rotation, and side-bending. Intensity of each exercise was varied by using different resistances of elastic tubing. Exercise balls and foam pads, provided in two levels of compliance (stiffness), were used for balance training. Four kinds of exercises were conducted: 1) standing on a foam pad, 2) moving the center of gravity in anterior/ posterior and medial/lateral directions while sitting on an exercise ball, 3) moving the center of gravity up and down while sitting on an exercise ball, and 4) moving the center of gravity in eight diagonal directions while standing on a foam pad.

\subsection{Measurements}

Body height, mass, BMI, fat, \%fat, muscle mass, functional fitness, and static and dynamic balance were measured before and after the intervention. All measurements were completed within one week before and after the intervention.

\subsubsection{Body composition}

Body fat and mass, and muscle mass were measured using a multi-frequency bioelectrical impedance analysis (X-SCAN plus; OWA Medical, JAPAN). Following measurement of body height and mass, body mass index (BMI) was computed as body mass $(\mathrm{kg})$ divided by the square of body height (m).

\subsubsection{Functional Fitness Test}

A battery of field tests, specifically developed for older adults, was used to assess the components of functional fitness. Using a standardized protocol, each test was conducted individually with the exception of the 12-min walk (TW). In this case, participants were instructed to set their own pace and to not walk with others. These tests have been shown to have content and construct validity as well as good test-retest reliability (Duncan et al., 1990; Rikli and Jones, 1999; Miotto et al., 1999; Rogers et 
al., 2003).

Upper-body strength was assessed using the 30-sec Arm Curl Test [AC] (Rikli and Jones, 1999). On a signal, participants were instructed to flex and extend the elbow of the dominant hand, lifting a weight (men: 8-pound dumbbell, women: 5-pound dumbbell) through the complete range of motion, as many times as possible in $30 \mathrm{sec}$. After demonstration by the tester, a practice trial of one or two repetitions was given, followed by one 30-s test trial. The score was the number of repetitions completed within $30 \mathrm{sec}$.

Lower-body strength was assessed using the 30-sec Chair Stand Test [CS] (Rikli and Jones, 1999). On a signal, participants rose to a full standing position from a chair and then returned to a seated position, and continued to complete as many full stands as possible in $30 \mathrm{sec}$. After demonstration by the tester, a practice trial of one to three repetitions was given, followed by one 30 -sec test trial. The score was the total number of stands executed correctly within $30 \mathrm{sec}$.

Balance and agility were assessed using the 8-foot Up and Go Test [UPGO] (Rikli and Jones, 1999) and Functional Reach Test [FR] (Duncan et al., 1999). To perform UPGO, participants were fully seated in a chair, hands on thighs and feet flat on the floor. On a signal, participants stood from the chair, walked as quickly as possible around a cone which was placed 8 feet $(2.44 \mathrm{~m})$ ahead of the chair, and returned to a fully seated position on the chair. Participants were told that this was a timed test and that the objective was to walk as quickly as possible (without running) around the cone and back to the chair. After demonstration by the tester, participants were given one practice trial and two test trials. The best performance time of the test trials was recorded in units of $0.1 \mathrm{sec}$. To perform FR, a scale (graduated in $\mathrm{cm}$ ) was hung from a wall at a height suitable for the participant. After demonstration by the tester, participants were given one practice trial and two test trials. The participant stood by the wall with feet together, hands clasped and both arms raised in front horizontally and held at the $0 \mathrm{~cm}$ level of the scale. On a signal, the participant leaned forward, moving the hands forward along the scale as far possible while keeping the heels in contact with the ground. Performance was assessed as the maximal distance the participant could reach forward beyond arms' length and without taking a step.

Upper-body flexibility was assessed using the Back Scratch Test [BS] (Rikli and Jones, 1999). Participants placed the preferred hand behind the same side shoulder with the forearm pronated and fingers extended. They placed the other hand behind the back, forearm supinated, reaching up in an attempt to touch or overlap the extended middle fingers of both hands. After demonstration by the tester, participants were asked to determine the preferred hand, and were given two practice trials, followed by two test trials. The score was the number of centimeters the middle fingers were short of touching (minus score) or overlapped each other (plus score). The best score of test trials was used to evaluate performance.

Lower-body flexibility was assessed using the Chair Sit and Reach Test [SR] (Rikli and Jones, 1999). Participants sat on the edge of a chair with one leg bent and the other leg extended straight in front with the heel on the floor. Without bending the knee, participants slowly reached forward, sliding the hands down the extended leg in an attempt to touch the toes. After demonstration by the tester, participants were asked to determine the preferred leg and were given two practice trials on that leg, followed by two test trials. The score was the number of centimeters short of reaching the toes (minus score) or reached beyond the toes (plus score). The best score of two test trials was used to evaluate performance.

Cardio-respiratory fitness was assessed by performing the 12-min Walk Test [TW] which assessed the maximum distance walked in $12 \mathrm{~min}$ around a 60-m rectangular course marked into 5-m segments (Takeshima et al., 1992; Yamauchi et al., 2005). The score was the total number of meters walked in 12 min. Predictive maximal oxygen uptake (predict $\mathrm{V}_{2}$ max) was calculated using an equation $(\mathrm{y}=0.025 \mathrm{x}-5.3, \mathrm{x}=\mathrm{TW})$ based on TW scores (Takeshima et al., 1992).

After the 12-wk the intervention, all measurements were repeated in each participant. All tests were conducted by the same tester during pre- and post-testing.

\subsubsection{Static and Dynamic Balance Test}

To measure static and dynamic balance, a Balance Master Platform System [Balance Master 8.0.2] (NeuroCom International, USA) was used 
(Rogers et al., 2003). Static balance measures are often taken while standing on different surfaces (firm surface or form pad) with the eyes open or closed. In this study, the Clinical Test of Sensory Interaction for Balance was conducted to test static balance and measure the influence of sensory input on balance. For this test, postural sway was evaluated via sway velocity (SV) while the participant stood quietly with eyes open (EO) on a firm surface, eyes closed (EC) on a firm surface, EO on a foam surface and EC on a foam surface. An index of composite mean for the four conditions (SVcomp) was used as a static balance parameter. The force platform was marked to maintain consistency in foot placement. For each stance, the participant stood with their eyes at the horizon and their arms at the sides in a neutral position. An anthropometric kit was used to measure standing height, foot length, and foot width. This information was used later to express the results relative to the height and base of support of each participant. Trials were $10 \mathrm{sec}$ in length and a trial was considered unsuccessful if the participant took a step or was unable to balance for the required time period without aid from a spotter.

Dynamic balance is the ability to anticipate changes and coordinate muscle activity in response to perturbations of stability (Rogers et al., 2003). Dynamic balance is also used during forward, sideways, and backward learning. In this study, dynamic balance was evaluated by performing the Limits of Stability (LOS) test on the same balance platform. The LOS is an assessment tool utilized to quantify the maximum distance an individual can intentionally displace their center of gravity, that is, lean in a specified direction without losing balance, stepping, or reaching for assistance (Newton, et al., 2001). Each participant stood on the platform viewing a monitor located to the front on which a cursor representing the center of pressure (COP) was viewed. The screen also showed nine boxes, one representing the centered COP position, and eight others representing targets spaced at 45-degree intervals in an oval shape representing $100 \%$ of the distance from the center position to the theoretical LOS. Each participant's age and height were used to calculate the theoretical LOS. Each participant was instructed to stand as still as possible while maintaining the COP cursor within the highlighted center target. When a black circle appeared in a designated outer LOS target, the participant was in- structed to move as quickly and accurately as possible so as to move the COP cursor to the designated target on a straight line, hold that position until termination of the trial $(10 \mathrm{sec})$, and then return the cursor to the center target (Rogers et al., 2003).

The MXE is the maximum distance the COP is displaced toward the target over the entire duration of the trial (Rogers et al., 2003). MXE is also expressed as a percentage of the distance to the target. In this study, indexes of composite mean of maximum excursion (MXEcomp) components, in the eight directions of LOS, were used for analysis.

Before the present study was conducted, the testretest reliability of balance testing $(n=13)$ was confirmed. Intra-class correlation coefficients [ICCs] for the Clinical Test of Sensory Interaction revealed a high level of reproducibility (ICC range = 0.88-0.90). The ICC values for Limits of Stability Test were 0.90-0.96 (Takeshima et al., 2013).

\subsection{Data analysis}

Data analysis was completed using the statistical software program SPSS for MAC (V.19.0, SPSS Inc., Chicago, IL). Absolute values were used for statistical analysis. However, when discussing differences between interventions on functional fitness measures, relative change was used to provide a clearer translation for the purpose of comparisons between measures with different units. Data were screened for outliers, and assumptions of normality and homoscedasticity. To reduce the potential influence of outliers on the statistical analysis, boxand-whiskers plots were used to identify outliers, which were subsequently eliminated prior to analysis. Each parameter was examined for normality using the Kolomogorov-Smirnov test. Assumptions of homogeneity of variance and sphericity were evaluated. Baseline group mean comparisons were performed using two-tailed independent $t$-tests. Comparisons between groups over time were evaluated using a two-way multivariate ANOVA with repeated measures. (ANOVA, Wilk's criterion). Group (EX, CN) served as the between-subject factor, while Time (pre- and post-test) served as the within-subject factor. Percent changes from pre to post were calculated from the differences in scores. Effect size [ES] was also calculated for each test. Cohen's definition of small, medium and large ES $(\mathrm{ES}=0.2,0.5$, and 0.8 , respectively) was used 
(Cohen, 1988). A probability value of less than 0.05 was considered statistically significant.

\section{Results}

\subsection{Normality and Assumptions}

Non-significant Kolomogorov-Smirnov tests indicated all parameters were normally distributed. In addition, histograms and normal Q-Q plots revealed normal distributions of parameters in all groups. Assumptions of homogeneity of variance and sphericity were evaluated and not violated.

\subsection{Pre-training Data}

No significant differences were noted at baseline for age, body height, mass, body fat, $\%$ fat, muscle mass and BMI between the two groups (Table 1). No significant baseline difference was noted in any measured functional fitness and balance parameter between the groups (Table 1).

\subsection{Training Data}

All participants continued a circuit exercise program using the FBES with no incidence of injury during the study. Mean attendance rate for the EX group was $92 \%$.

Repetitions, force, and RPE during resistance exercise progressively increased during the second and third stages (Table 2). The repetitions and force varied by exercise, but subjective perceived exertion increased overall from easy to somewhat hard.

The mean heart rate and relative exercise intensity (\%HRR) during the exercise sessions was $115 \pm 14$ bpm and $50.1 \%$, and $123 \pm 14 \mathrm{bpm}$ and $60.1 \%$ in the second and third stages, respectively (Table 3).

\subsection{Training Effect Data}

After 12-wk of exercise training, there were no significant interactions (group $\times$ time) for body mass, and BMI (Table 4).

After 12-wk of training, there were significant interactions (group $\times$ time) for $\mathrm{AC}, \mathrm{TW}$, predict $\dot{\mathrm{O}}_{2} \max$, UPGO, BS, and SR (Table 4). However, significance interactions were not found for CS, FR, SVcomp, and MXEcomp (Table 4).

\section{Discussion}

This study evaluated the effects of a 12 -wk circuit exercise program consisting of concurrent aerobic, resistance, flexibility, and balance training using the FBES on fitness in community-dwelling older women. Based on the results of the present study, it appears that incorporating aerobic, resistance, and flexibility training via the FBES is an effective means to improve multiple aspects of fitness with a single exercise program when performed at a moderate intensity. Specifically, the present study demonstrated increases in upper body strength $(\mathrm{AC}=12.6 \%)$, mobility $(\mathrm{UPGO}=-8.7 \%$ ), cardiorespiratory fitness $\left(\mathrm{TW}=5.4 \%\right.$ and predict $\left.\dot{\mathrm{V}} \mathrm{O}_{2} \max =6.3 \%\right)$, and flexibility $(\mathrm{SR}=24.3 \%$; $\mathrm{BS}=271.4 \%)$. Based on these results, it appears that this type of circuit exercise can serve as a well-rounded exercise intervention for older adults. Although the FBES depends upon subjective maximal effort when using elastic resistance, and it is difficult to determine if participants were training at this level of intensity, it was sufficient to improve upper body strength (i.e., AC). Recently, Yoon et al. (2016) suggested that highspeed power training using elastic resistance improved physical performance and muscle strength in older women with mild cognitive impairment. Therefore, future studies using the FBES should evaluate the velocity and power developed during exercise as a way to determine intensity. Although various components of fitness improved using the FBES, improvements in static and dynamic balance were not observed. These results are similar to those from larger, more expensive hydraulic exercise machines (Takeshima et al., 2004).

Our previous study (2004) demonstrated that circuit training using hydraulic resistance machines increased oxygen uptake at lactate threshold (29\%) and at peak $\dot{\mathrm{VO}}_{2}(15 \%)$ measured using an incremental cycle ergometer. We did not directly measure $\dot{\mathrm{V}} \mathrm{O}_{2}$ max in this study; rather we predicted it based on TW results using the equation developed by Takeshima et al. (1992). Although comparatively less than the improvements in peak $\dot{\mathrm{VO}}_{2}$ of our previous study, predict $\mathrm{V}_{2}$ max for EX did significantly improve $(6.3 \%)$ with a medium $(0.56)$ ES. Muscular strength after 12-wk of PACE training increased for knee extension, knee flexion, back extension and flexion, chest pull and press, shoulder press and pull, and leg press. Body fat also de- 
Table 2 Repetition, force, and RPE (rating of perceived exertion) during resistance exercise sessions using the floor-based exercise station (FBES).

\begin{tabular}{|c|c|c|c|}
\hline Movement & First stage (1-4 wk) & Second stage (5-8 wk) & Third stage (9-12 wk) \\
\hline \multicolumn{4}{|l|}{ Arm curl } \\
\hline repetition & - & $20.1 \pm 4.4$ & $26.4 \pm 6.8$ \\
\hline force $(\mathrm{kg})$ & - & $3.1 \pm 0.3$ & $3.4 \pm 0.8$ \\
\hline RPE & - & $14(15-12)$ & $14(15-12)$ \\
\hline Squat & - & & \\
\hline repetition & - & $17.1 \pm 6.3$ & $18.7 \pm 5.3$ \\
\hline force $(\mathrm{kg})$ & - & $3.7 \pm 1.1$ & $8.4 \pm 3.3$ \\
\hline RPE & - & $12(14-10)$ & $13(15-10)$ \\
\hline Upright Row & - & & \\
\hline repetition & - & $25.3 \pm 7.9$ & $25.1 \pm 6.5$ \\
\hline force $(\mathrm{kg})$ & - & $3.7 \pm 1.2$ & $5.7 \pm 0.5$ \\
\hline RPE & - & $13(15-11)$ & $13(15-12)$ \\
\hline Lateral Rise & - & & \\
\hline repetition & - & $18.9 \pm 6.8$ & $23.2 \pm 7.9$ \\
\hline force $(\mathrm{kg})$ & - & $2.0 \pm 0.8$ & $2.3 \pm 0.9$ \\
\hline RPE & - & $13(15-11)$ & $13(15-11)$ \\
\hline Elbow Extension & - & & \\
\hline repetition & - & $23.4 \pm 7.4$ & $25.8 \pm 8.5$ \\
\hline force $(\mathrm{kg})$ & - & $2.7 \pm 1.2$ & $2.9 \pm 0.7$ \\
\hline RPE & - & $13(15-11)$ & $13(15-11)$ \\
\hline Low Back Extension & - & & \\
\hline repetition & - & $17.5 \pm 6.0$ & $15.8 \pm 5.0$ \\
\hline force $(\mathrm{kg})$ & - & $8.3 \pm 1.4$ & $8.6 \pm 1.4$ \\
\hline RPE & - & $12(13-10)$ & $13(16-11)$ \\
\hline Trunk Rotation & - & & \\
\hline repetition & - & $24.2 \pm 6.6$ & $29.1 \pm 15.1$ \\
\hline force $(\mathrm{kg})$ & - & $1.7 \pm 0.8$ & $3.2 \pm 2.0$ \\
\hline RPE & - & $12(14-11)$ & $12(16-11)$ \\
\hline Side Bend & - & & \\
\hline repetition & - & $21.1 \pm 5.6$ & - \\
\hline force $(\mathrm{kg})$ & - & $4.0 \pm 1.3$ & - \\
\hline RPE & - & $12(15-11)$ & - \\
\hline
\end{tabular}

Note: Repetion: number of repetitions; RPE: Rating of Perceved Exersion, displays the median (max-min); —: no record 
Table 3 Heart rate and exercise intensity during circuit exercise training program using the floor-based exercise station (FBES).

\begin{tabular}{|c|c|c|c|c|c|c|c|}
\hline & \multicolumn{3}{|c|}{ Aerobic exercise (dance) } & \multicolumn{3}{|c|}{ Resistance \& Balance exercise } & \multirow[t]{2}{*}{ Total } \\
\hline \multicolumn{7}{|c|}{ Second stage (5-8 wk) } & \\
\hline Time & Mean \pm SD & $\max$ & $\min$ & Mean \pm SD & $\max$ & $\min$ & Mean \pm SD \\
\hline 0 to $10 \mathrm{~min}$ & $109 \pm 19$ & 138 & 81 & $109 \pm 18$ & 126 & 77 & $109 \pm 19(43 \%$ HRR $)$ \\
\hline 10 to $20 \mathrm{~min}$ & $114 \pm 19$ & 139 & 87 & $117 \pm 19$ & 136 & 79 & $116 \pm 15(52 \%$ HRR $)$ \\
\hline 20 to $30 \mathrm{~min}$ & $122 \pm 21$ & 156 & 88 & $119 \pm 22$ & 144 & 82 & $120 \pm 22(57 \%$ HRR $)$ \\
\hline Total $30 \mathrm{~min}$ & $115 \pm 19$ & 156 & 88 & $115 \pm 20$ & 144 & 82 & $115 \pm 20(51 \%$ HRR $)$ \\
\hline \multicolumn{8}{|c|}{ Final Stage (9-12 wk) } \\
\hline 0 to $10 \mathrm{~min}$ & $117 \pm 20$ & 137 & 79 & $115 \pm 20$ & 142 & 89 & $116 \pm 20(53 \%$ HRR $)$ \\
\hline 10 to $20 \mathrm{~min}$ & $122 \pm 19$ & 140 & 90 & $123 \pm 19$ & 144 & 99 & $123 \pm 19(61 \%$ HRR $)$ \\
\hline 20 to $30 \mathrm{~min}$ & $128 \pm 22$ & 151 & 90 & $129 \pm 24$ & 147 & 95 & $128 \pm 23(67 \%$ HRR $)$ \\
\hline Total $30 \mathrm{~min}$ & $122 \pm 20$ & 151 & 90 & $123 \pm 21$ & 147 & 99 & $122 \pm 21(60 \%$ HRR $)$ \\
\hline
\end{tabular}

Note: HRR: heart rate reserve Karvonen method

Table 4 Effect of Exercise using the floor-based exercise station (FBES).

\begin{tabular}{|c|c|c|c|c|c|c|c|c|c|c|}
\hline \multirow{2}{*}{\multicolumn{2}{|c|}{ parameters }} & \multicolumn{4}{|c|}{ Exercise (EX) group } & \multicolumn{4}{|c|}{ Control group (CN) } & \multirow{2}{*}{$\begin{array}{c}\text { Interaction } \\
\text { F-value } \\
\text { (group } \times \text { time) }\end{array}$} \\
\hline & & Pre & Post & $\begin{array}{c}\text { Change } \\
(\%)\end{array}$ & ES & Pre & Post & $\begin{array}{c}\text { Change } \\
(\%)\end{array}$ & ES & \\
\hline \multirow{5}{*}{$\begin{array}{l}\text { Body } \\
\text { Composition }\end{array}$} & Body Mass (kg) & $49.4 \pm 5.5$ & $49.0 \pm 5.5$ & -0.8 & 0.07 & $50.6 \pm 6.6$ & $50.5 \pm 6.5$ & -0.2 & 0.02 & 0.60 (N.S.) \\
\hline & $\%$ fat $(\%)$ & $29.5 \pm 3.8$ & $28.5 \pm 3.7$ & -3.4 & 0.26 & $28.5 \pm 3.7$ & $29.4 \pm 3.7$ & 3.2 & 0.24 & $37.08^{*}$ \\
\hline & Fat $(\mathrm{kg})$ & $14.7 \pm 3.0$ & $14.1 \pm 2.9$ & -4.1 & 0.20 & $14.6 \pm 3.3$ & $15.0 \pm 3.3$ & 2.7 & 0.12 & $25.36^{*}$ \\
\hline & LBM (kg) & $34.7 \pm 3.4$ & $34.9 \pm 3.4$ & 0.6 & 0.06 & $36.0 \pm 4.1$ & $35.5 \pm 3.8$ & -1.4 & 0.12 & $9.19^{*}$ \\
\hline & BMI $\left(\mathrm{kg} / \mathrm{cm}^{2}\right)$ & $21.8 \pm 2.2$ & $21.6 \pm 2.1$ & -0.9 & 0.09 & $22.0 \pm 2.3$ & $22.0 \pm 2.2$ & 0 & 0.00 & 0.76 (N.S.) \\
\hline \multirow{8}{*}{$\begin{array}{l}\text { Functional } \\
\text { Fitness }\end{array}$} & $\mathrm{AC}$ (times/30 sec) & $26.2 \pm 3.7$ & $29.5 \pm 4.3$ & 12.6 & 0.89 & $25.7 \pm 5.0$ & $26.6 \pm 4.5$ & 3.5 & 0.18 & $6.74^{*}$ \\
\hline & CS (times/30 sec) & $28.9 \pm 5.5$ & $31.7 \pm 3.9$ & 9.7 & 0.51 & $27.3 \pm 6.0$ & $28.5 \pm 6.5$ & 4.4 & 0.20 & 2.54 (N.S.) \\
\hline & UPGO (sec) & $4.35 \pm 0.56$ & $3.97 \pm 0.45$ & -8.7 & 0.68 & $4.24 \pm 0.62$ & $4.31 \pm 0.58$ & 1.7 & 0.11 & $16.15^{*}$ \\
\hline & FR $(\mathrm{cm})$ & $32.3 \pm 5.0$ & $33.2 \pm 4.1$ & 2.8 & 0.18 & $31.9 \pm 2.9$ & $30.9 \pm 2.8$ & -3.1 & 0.35 & 1.72 (N.S.) \\
\hline & $\mathrm{SR}(\mathrm{cm})$ & $18.1 \pm 9.1$ & $22.5 \pm 9.3$ & 24.3 & 0.48 & $19.6 \pm 8.4$ & $18.3 \pm 8.3$ & -6.6 & 0.16 & $35.54^{*}$ \\
\hline & $\mathrm{BS}(\mathrm{cm})$ & $0.7 \pm 7.9$ & $2.6 \pm 7.3$ & 271.4 & 0.24 & $-1.5 \pm 13.1$ & $-2.0 \pm 12.5$ & 33.3 & 0.04 & $5.86^{*}$ \\
\hline & TW (m) & $1162 \pm 112$ & $1225 \pm 108$ & 5.4 & 0.59 & $1131 \pm 83$ & $1156 \pm 92$ & 2.2 & 0.30 & $4.19^{*}$ \\
\hline & Predic $\dot{V}_{2} \max$ & $23.8 \pm 2.7$ & $25.3 \pm 2.7$ & 6.3 & 0.56 & $22.7 \pm 2.4$ & $23.3 \pm 2.7$ & 2.6 & 0.25 & $5.14^{*}$ \\
\hline \multirow{2}{*}{ Balance } & SVcomp (deg/sec) & $0.82 \pm 0.25$ & $0.85 \pm 0.18$ & 3.7 & 0.12 & $0.81 \pm 0.18$ & $0.73 \pm 0.12$ & -9.9 & 0.45 & 3.77 (N.S.) \\
\hline & MXEcomp (\%) & $93.4 \pm 5.6$ & $93.8 \pm 4.8$ & 0.4 & 0.07 & $92.4 \pm 4.0$ & $95.7 \pm 4.1$ & 3.6 & 0.83 & 2.22 (N.S.) \\
\hline
\end{tabular}

Note: LBM: lean body mass; BMI: body mass index (BMI) was computed as body weight (kg) divided by the square of height (m);

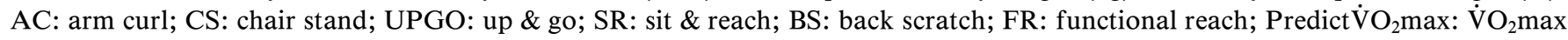
was predicted by an equation from 12-min walk; TW: 12-min walk; SVcomp: composite sway velocity scores were calculated based on each sway velocity condition; EPEcomp: composite endpoint excursion scores were calculated based on movements toward all 8 targets appearing around a center square at $0,45,90,135,180,225,270$, and 315 degrees; MXE: composite maximum excursion scores were calculated based on movements toward all 8 targets appearing around a center square at $0,45,90,135,180,225,270$, and 315 degrees; N.S., not significant. 
creased and HDL cholesterol increased. Although hydraulic resistance machines and the FBES both improved aerobic fitness and strength, different tests were used to measure parameters of fitness in these two studies so it is difficult to directly compare the effects of the two interventions.

The FBES is an inexpensive, small, portable device that allows multiple exercises to be performed. These attributes have benefits for facilities with little storage space where larger equipment would be prohibitive. In addition, attendance of participants in the current study was $92 \%$. This high rate of participation was likely due to the fact that participants enjoyed the group-based nature of the program as it gave them opportunities for social interaction and they also found the FBES easy to use. These qualities suggest that this program could be sustainable as older adults would be more likely to participate in a program such as this rather than exercising alone on large, complicated machines.

Although CS did not significantly improve [pre $28.9 \pm 5.5$, post $31.7 \pm 3.9$ times/30 sec, $9.7 \%$ ], the $\mathrm{ES}$ was medium $(\mathrm{ES}=0.51)$. The lack of significant improvements may be due to the nature of the other exercises performed in the circuit program. Specifically, the aerobic and balance exercises also involved the lower-body and this may have limited the intensity achieved during the squat exercise. GineGarriga et al. (2013) showed that a 12-week circuit training program improved physical function in frail older adults. However, this program consisted of only two types of exercise: functional balance and lower-body strength exercises. As the specificity of exercise becomes more specific, targeted physiologic systems are more likely to improve. Further research is needed to determine if adding additional lower-body resistance exercises to the FBES circuit training program results in improvements in CS.

A novel component of this circuit training program was the incorporation of balance exercises. However, balance parameters did not improve as a result of training. Although specific guidelines for aerobic and resistance exercise have been published (ACSM, 2009; 2011), the evidence to equally support dose-response relationships for balance training does not exist (Howe et al., 2007). Exercise programs specifically targeting the control systems (e.g., visual, vestibular, somatosensory and musculoskeletal) that monitor balance have led to improvements in the postural balance of older adults and most likely reduces their risk of falls (Islam et al., 2004; Rogers et al., 2005; Narita et al., 2014). It also appears that such training is more effective in improving balance compared to more general exercise programs consisting of only aerobic, strength, or flexibility exercises (Rogers et al., 2005). For example, Islam and colleagues (2004) evaluated older adults participating in a 12 -wk customized balance training program utilizing the same modalities used in the present study (i.e., foam stability trainers) and found improvements in LOS. This difference in results is likely attributable to the much larger training volume for balance exercises where nearly 50 hours of balance-specific exercises were performed compared to the relatively small amount performed in the current study. Further research is needed to determine how balance can be enhanced as part of a 12-wk circuit training program.

Although further study is needed, the FBES is a relatively inexpensive means by which circuit training can be conducted in group-settings with little requirement for space. This study supports the efficacy of this product for older adults and implementation of this program could be feasible in retirement communities, senior centers, as well as in home settings.

\section{Acknowledgments}

The authors are grateful to the subjects who participated in this study.

\section{References}

American College of Sports Medicine. (2009). Position stand: Progression models in resistance training for healthy adults. Med. Sci. Sports Exerc., 41: 687-708.

American College of Sports Medicine. (2009). Exercise and physical activity for older adults- Position stand. Med. Sci. Sports Exerc., 41: 1510-1530.

American College of Sports Medicine. (2011). Quantity and quality of exercise for developing and maintaining cardiorespiratory, musculoskeletal, and neuromotor fitness in apparently healthy adults: Guidance for prescribing exercise. Med. Sci. Sports Exerc., 43: 1334-1359.

Borg, G. (1982). Psychophysical bases of perceived exertion. Med. Sci. Sports Exerc., 14: 377-381.

Cohen, J. (1988). Statistical Power Analysis for the Behavioral Sciences (2nd ed.) Hillsdale, New Jersey: Lawrence Erlbaum. Duncan, P. W., Weiner, D. K., Chandler. J., and Studenski, S. (1990). Functional reach: a new clinical measure of balance. J. Gerontol., 45: M192-M197.

Gine-Garriga, M., Guerra, M., and Unnthan, V. B. (2013). The effect of functional circuit training on self-reported fear of falling and health status in a group of physically frail older individuals: a ramdomized controlled trial. Aging Clin. Exp. Res., Jun; 25(3): 329-36. doi: 10.1007/s40520-013-0048-3. 
Epub 2013 May 17.

Howe, T. E., Rochester, L., Jackson, A., Banks, P. M., and Blair, V. A. (2007). Exercise for improving balance in older people. Cochrane Database of Sys. Rev., Issue 4, CD004963.

Islam, M. M., Nasu, E., Rogers, M. E., Koizumi, D., Rogers, N. L., and Takeshima, N. (2004). Effects of combined sensory and muscular training on balance in older adults. Prev. Med., 39: 1148-1155.

Izquierdo, M. I., Ibañez, J., Hakkinen, K., Kraemer, W. J., Larrión, J. L., and Gorostiaga, E. M. (2004). Once weekly combined resistance and cardiovascular training in healthy older men. Med. Sci. Sports Exerc., 36: 435-43.

Karvonen, M., Kentala, K., and Mustala, O. (1957). The effects of training on heart rate: a longitudinal study. Ann. Med. Exp. Biol. Fenn., 35: 307-315.

Miotto, J. M., Chodzko-Zajko, W. J., Reich, J. L., and Supler, M. M. (1999). Reliability and validity of the Fullerton Functional Fitness Test: an independent replication study. J. Aging Phys. Activ., 7: 339-353.

Narita, M., Islam, M. M., Rogers, M. E., Koizumi, D., and Takeshima, N. (2015). Effects of customized balance exercises on older women whose balance ability has deteriorated with age. J. Women Aging, 27(3): 237-250.

National Institute on Aging. https: //www.nia.nih.gov/about/ nia-timeline

Newton, R. (2001). Validity of the multi-directional reach test: a practical measure for limits of stability in older adults. $\mathrm{J}$. Gerontol. Series A. Biol. Sci. Med. Sci., 56: M248-M252.

Rikli, R. E. and Jones, C. J. (1999). Development and validation of a functional fitness test for community-residing older adults. J. Aging Phys. Activ., 7: 129-161.

Rogers, M. E., Rogers, N. L., Takeshima, N., and Islam, M. M. (2003). Methods to evaluate and improve the physical parameters associate with fall risk in older adults. Prev. Med., 36: 255-264.

Rogers, M. E., Rogers, N. L., and Takeshima, N. (2005). Balance training in older adults. J. Aging Health, 8(4): 1475-486.

Takeshima, N., Islam, M. M., Rogers, M. E., Rogers, N. L., Sengoku, N., Koizumi, D., Kitabayashi, Y., Imai, A., and Naruse, A. (2013). Effects of Nordic walking compared to conventional walking and band-based resistance exercise on fitness in older adults. J. Sports Sci. Med., 12: 422-430.

Takeshima, N., Rogers, M. E., Islam, M. M., Yamauchi, Y., Watanabe, E., and Okada, A. (2004). Effects of concurrent aerobic and resistance circuit exercise training on fitness in older adults. Eur. J. Appl. Physiol., 93: 173-182.

Takeshima, N., Rogers, N. L., Rogers, M. E., Islam, M. M., Koizumi, D., and Lee, S. (2007). Functional fitness gain varies in older adults depending on exercise mode. Med. Sci. Sports Exerc., 39: 2036-2043.

Takeshima, N., Tanaka, K., Kobayashi, F., Sumi, K., Watanabe, T., and Kato, T. (1992). Validity of the maximal aerobic capacity estimated from submaximal cycling exercise and field performance tests in the elderly. Japan J. Physi. Fit and Sports Med., 41: 295-303 (in Japanese).

Wood, R. H., Reyes, R., Welsch, M. A., Favaloro-Sabatier, J., Sabatier, M., Matthew, L. C., Johnson, L. G., and Hooper, P. F. (2001). Concurrent cardiovascular and resistance training in healthy older adults. Med. Sci. Sports Exerc., 33: 1751-1758.

Yamauchi, T., Islam, M. M., Koizumi, D., Rogers, M. E., Rogers, N. L., and Takeshima, N. (2005). Effect of homebased well-rounded exercise in community-dwelling older adults. J. Sports Sci. Med., 4: 563-571.

Yoon, D. H., Kang, D., Kim, S. M., Song, H. S., and Song, W. (2016). Effect of elastic band-based high speed power training on cognitive function, physical performance and muscle strength in older women with mild cognitive impairment. Geriati. Gerontol. Int., 1 10. doi: 10.1111/ggi. 12784. [Epub ahead of print]

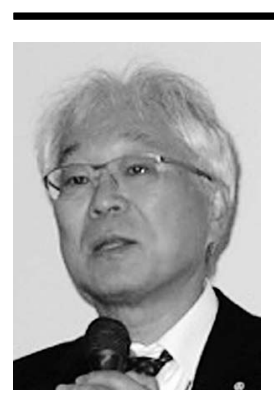

Name:

Nobuo Takeshima

\section{Affiliation:}

Department Chairman \& Professor in Asahi University, and a Fellow of Active Aging Association (a Non-Profit Organization, made by June 2006)

\section{Address:}

Department of Health and Sports Sciences, School of Health Sciences, Asahi University, Mizuho, Gifu 501-0296, JAPAN

\section{Brief Biographical History:}

2017- Department of Health and Sports Sciences, School of Health Sciences, Asahi University, 1851 Hozumi, Mizuho, Gifu 501-0296 JAPAN

2011-2017 Laboratory of Exercise Gerontology, National Institute of Fitness and Sports in Kanoya, Kagoshima, JAPAN 1980-2011 Laboratory of Exercise Gerontology, Graduate School of Natural Sciences, Nagoya City University, Nagoya, JAPAN

\section{Main Works:}

-Exercise Gerontology, Health Promotion

Membership in Learned Societies:

- Japan Society of Physical Education and Sports Sciences, American College of Sports Medicine

\section{Research interests:}

-Exercise prescription, Well-rounded exercise program, Older adults 